\title{
Can a Transport Model Predict Inverse Signatures in Lithium Metal Batteries Without Modifying Kinetics?
}

Maitri Uppaluri ${ }^{1, *}$, Akshay Subramaniam ${ }^{2, *}$, Lubhani Mishra ${ }^{3}$, Vilayanur Viswanathan ${ }^{4}$, Venkat R. Subramanian ${ }^{1,3, * *, z}$

${ }^{1}$ Materials Science and Engineering Program, Texas Materials Institute, The University of Texas at Austin, Austin, TX 78712, USA

${ }^{2}$ Department of Chemical Engineering, University of Washington, Seattle, WA 98195, USA

${ }^{3}$ Walker Department of Mechanical Engineering \& Material Science Engineering, Texas Materials Institute, The University of Texas at Austin, Austin, Texas 78712, USA ${ }^{4}$ Pacific Northwest National Laboratory, 902 Battelle Blvd., Richland, WA 99354, USA

*Electrochemical Society Student Member

**Electrochemical Society Fellow

zEmail:venkat.subramanian@utexas.edu 


\begin{abstract}
In this study, a one-dimensional transport model is developed and analyzed, which predicts the inverse signatures obtained in lithium metal batteries. This simple approach's ability to predict inverse signatures stems from the competing interplay between moving boundary rates and mass transfer limitations to yield inverse signatures without modifying the kinetics that describes the deposition and dissolution of lithium on the electrode surface. The numerical scheme used for the present model simulations is presented in detail which has been further used to study the effect of design parameters on the prevalence and strength of inverse signatures. It was found that the proposed model and the analysis is more pertinent to symmetric cells, commonly used for in-depth fundamental studies.
\end{abstract}




\section{Introduction}

Over the recent years, the employment of lithium metal as the anode material in lithium batteries has been gaining widespread momentum due to its highly favorable electrochemical characteristics, viz., high theoretical capacity $\left(3,860 \mathrm{mAh} \mathrm{g}^{-1}\right)$ and low electrochemical potential $\left(-3.040 \mathrm{~V}\right.$ vs. standard hydrogen electrode). ${ }^{1}$ These features enable batteries with lithium metal anodes to have higher energy density and specific energy in comparison to the conventional graphite anodes found in commercial lithium-ion batteries today. These advantages are paving the path of lithium metal batteries towards commercialization by their adoption in important applications like electric vehicles and grid-scale energy storage. However, the implementation of lithium metal anodes poses several shortcomings concerning safety and cyclability. ${ }^{2}$ The state-ofthe-art for lithium metal batteries suggests that one of the primary hurdles in their commercial usage is the deposition of lithium on the electrode surface. The reactivity of Li metal with the electrolyte leads to the formation of the solid electrolyte interphase (SEI) which grows, becomes unstable and increases the impedance of the cell. ${ }^{1-3}$ The growth of needle-like dendrites can punch into the separator and lead to short-circuiting of the cell. Dendritic electrodeposition on the lithium metal surface leads to the accumulation of dead lithium on the electrode surface causing reduced lifetime. ${ }^{1,2,4}$ Thus, these challenges incontrovertibly pique the research efforts towards a more detailed understanding in order to enhance the applicability of this advantageous technology.

Several mathematical models construing the reaction and transport mechanisms for electrodeposition have been explored extensively in the past. The initial models depicted the dendrite formation as a result of electrodeposition to be diffusion-limited. ${ }^{5,6}$ Chazalviel $^{7}$ modeled the formation of dendrites at high electric fields and found that the growth of ramified deposits is 
due to the creation of a space charge near the electrode surface, where the assumption of electroneutrality is violated. Monroe et al. ${ }^{8}$ proposed a modified form of the Butler-Volmer kinetics which characterizes the lithium deposition by accounting for the changes in the surface energy associated with the formation and growth of needle-like dendrites.

The voltage response of a lithium metal cell has a characteristic nature where the voltage drops and rises, termed as "inverse signatures". Wood et al. ${ }^{9,10}$ suggested that the deposition and dissolution of lithium are inscribed onto the voltage response of the cell characterized by unique signatures due to the relative dominance among the competing mechanisms at the electrode surface. They also proposed a model to obtain the voltage signature using a modified form of the kinetics expression and including the changes in surface area associated with pitting, $\mathrm{Li}^{+}$removal from the bulk and dendrite dissolution at one electrode, and nucleation and growth of dendrites at the other electrode in a symmetric cell. The transition from one process to another is captured by changes in the overpotential which, in turn, invokes these inverse signatures in the voltage response. It was also demonstrated that different types of dendritic formation on the electrode surface, namely, mossy, needle and fractural dendrites result in distinct indicative electrochemical signatures. Two mechanisms were proposed by Bai et al. ${ }^{11}$ to interrelate the change in morphology of lithium deposits with the voltage transition. The formation of dendrites stayed reaction-limited with mossy lithium growth from the root as the electrolytic salt concentration approached zero at Sand's capacity. The voltage experienced a spike at this juncture due to a shift in the mechanism to transport-limited growth of dendritic structures.

It can be ascertained that several factors affect the formation and the growth of the deposits on the electrode, and thus have an influence on the resulting voltage signature. Furthermore, the applied current density during charging/discharging of the cell also impacts the morphology of dendritic 
deposits. ${ }^{12}$ Previous studies examine dendrite growth at current densities higher than the mass transfer limiting current density. ${ }^{6,7}$ High current densities can deplete the ion concentration near the electrode and create a space charge in the vicinity of the electrode that facilitates deposition. ${ }^{7}$ Dendrites are observed at lower current densities as well, but with different morphologies and after extended times. ${ }^{10,13}$ For a given cell, the study of dendrite growth with current density is performed with fractions ranging from 0.05 to 0.95 of the mass transfer limiting current density for that cell configuration. ${ }^{8,11,14}$ Monroe et al. ${ }^{8}$ showed that lowering the current density slows down the growth of the deposit.

Besides, the nature of the electrolyte and the salt used in the cell play a role in the initial nucleation, growth and the morphology of these dendrites. High salt concentration in the electrolyte increases the critical current density for the dendrite formation, thus suppressing their formation. ${ }^{2,15}$ Frenck et al. ${ }^{14}$ studied the influence of the shear modulus of the electrolyte and the current density on the morphology of the dendrites.

The formulation of a numerical model to predict these signatures in lieu of the delicate experiments has far-reaching implications and can be extensively used to elucidate the electrodeposition of lithium. Our previous attempt to formulate a model predicts the characteristic decrease and increase ("inverse") in the voltage response for a given current density by using a piecewise function for the exchange current density to accommodate for the changes in reaction pathways observed during lithium deposition. ${ }^{16}$ The present work not only proposes a one-dimensional model for the deposition of lithium on the electrode in a lithium symmetric cell based on the transport of the lithium ions within the cell but also represses the need for any modification in the kinetics. The model treats the lithium electrodeposition as a moving interface, where the velocity of the interface is defined using Faraday's law. ${ }^{17}$ It is worthwhile to mention that this method is 
efficient to obtain the inverse signatures, i.e., typical characteristics of lithium deposition and dissolution, without accounting for the changes in the kinetics to capture the nucleation and growth of the deposits. In order to check for the robustness and to demonstrate that a transport model can produce unique voltage signatures with different operating conditions and design parameters, the present model is tested across different parameters to study the unique 'transport' induced voltage signatures for different cases in the typical lithium symmetric cells.

\section{Model Description}

Figure 1 shows the schematic of a characteristic lithium symmetric cell, where lithium is dissolved from one electrode and deposited onto the surface of the other lithium metal electrode on the application of current. The electrolyte considered is a binary electrolyte consisting of $\mathrm{Li}^{+}$and an anion in the solution with constant diffusivities of the ionic species. The governing equations and boundary conditions describing the flux of the species in the electrolyte and the conversation of the species are derived in detail in Subramaniam et al. ${ }^{16}$ and are reiterated below.

The mass transport flux between the two electrodes for the each of the ionic species in terms of diffusion and migration can be written as:

$$
\begin{aligned}
& \mathrm{N}_{1}=-\mathrm{D}_{1} \frac{\partial \mathrm{c}_{1}}{\partial \mathrm{x}}-\mathrm{z}_{1} \mathrm{u}_{1} \mathrm{Fc}_{1} \frac{\partial \phi}{\partial \mathrm{x}} \\
& \mathrm{N}_{2}=-\mathrm{D}_{2} \frac{\partial \mathrm{c}_{2}}{\partial \mathrm{x}}-\mathrm{z}_{2} \mathrm{u}_{2} \mathrm{Fc}_{2} \frac{\partial \phi}{\partial \mathrm{x}}
\end{aligned}
$$

where $\mathrm{N}, \mathrm{D}, \mathrm{x}, \mathrm{z}, \mathrm{u}, \mathrm{F}$, and $\phi$ are the total flux $\left(\mathrm{mol} / \mathrm{m}^{2} \mathrm{~s}\right)$, diffusivity $\left(\mathrm{m}^{2} / \mathrm{s}\right)$, concentration $\left(\mathrm{mol} / \mathrm{m}^{3}\right)$, position along the length of the cell (m), charge of the ion, ionic mobility, Faraday's constant $(\mathrm{C} / \mathrm{mol})$ and electrostatic potential $(\mathrm{V})$, respectively. The subscripts 1 and 2 represent the cation and anion, respectively. 
The material balance of the ionic species can be written using the Nernst-Planck equation in one dimension as:

$$
\begin{aligned}
& \frac{\partial \mathrm{c}_{1}}{\partial \mathrm{t}}=-\frac{\partial \mathrm{N}_{1}}{\partial \mathrm{x}} \\
& \frac{\partial \mathrm{c}_{2}}{\partial \mathrm{t}}=-\frac{\partial \mathrm{N}_{2}}{\partial \mathrm{x}}
\end{aligned}
$$

The ionic mobility of a species can be related to its diffusivity by using the Nernst-Einstein relation.

$$
\begin{aligned}
& \mathrm{u}_{1}=\frac{\mathrm{D}_{1}}{\mathrm{RT}} \\
& \mathrm{u}_{2}=\frac{\mathrm{D}_{2}}{\mathrm{RT}}
\end{aligned}
$$

where $\mathrm{R}$ is the gas constant $(\mathrm{J} / \mathrm{mol} \mathrm{K})$, and $\mathrm{T}$ is the absolute temperature $(\mathrm{K})$.

Substituting these expressions for the ionic mobilities in Equations 3 and 4, we obtain the following expressions for the material balance for the ionic species.

$$
\begin{aligned}
& \frac{\partial \mathrm{c}_{1}}{\partial \mathrm{t}}=-\frac{\partial}{\partial \mathrm{x}}\left(-\mathrm{D}_{1} \frac{\partial \mathrm{c}_{1}}{\partial \mathrm{x}}-\frac{\mathrm{D}_{1} \mathrm{~F}}{\mathrm{RT}} \mathrm{c}_{1} \frac{\partial \phi}{\partial \mathrm{x}}\right) \\
& \frac{\partial \mathrm{c}_{2}}{\partial \mathrm{t}}=-\frac{\partial}{\partial \mathrm{x}}\left(-\mathrm{D}_{2} \frac{\partial \mathrm{c}_{2}}{\partial \mathrm{x}}+\frac{\mathrm{D}_{2} \mathrm{~F}}{\mathrm{RT}} \mathrm{c}_{2} \frac{\partial \phi}{\partial \mathrm{x}}\right)
\end{aligned}
$$

It should be noted that the electroneutrality assumption is valid for the domain of interest.

For a binary electrolyte, the electroneutrality assumption is given by: 


$$
\sum_{\mathrm{i}} \mathrm{z}_{\mathrm{i}} \mathrm{c}_{\mathrm{i}}=0
$$

For a binary electrolyte where the charge of the cation $\left(\mathrm{Li}^{+}\right), \mathrm{z}_{1}=1$, and the anion, $\mathrm{z}_{2}=1$, the electroneutrality assumption gives $\mathrm{c}_{1}=\mathrm{c}_{2}$.

Now, assuming $c_{1}=c_{2}=c$, the expressions for the conservation of the species, Equations 7 and 8 can be rewritten in terms of $\mathrm{c}$ as:

$$
\begin{aligned}
& \frac{\partial c}{\partial t}=-\frac{\partial}{\partial x}\left(-D_{1} \frac{\partial c}{\partial x}-\frac{D_{1} F}{R T} c \frac{\partial \phi}{\partial x}\right) \\
& \frac{\partial c}{\partial t}=-\frac{\partial}{\partial x}\left(-D_{2} \frac{\partial c}{\partial x}-\frac{D_{2} F}{R T} c \frac{\partial \phi}{\partial x}\right)
\end{aligned}
$$

An effective diffusivity, $\mathrm{D}_{\text {eff, }}$ is considered for the binary electrolyte, where

$$
D_{\text {eff }}=\frac{2 D_{1} D_{2}}{D_{1}+D_{2}}
$$

For a binary electrolyte, the applied current density can be related to the fluxes as:

$$
\mathrm{F} \sum_{\mathrm{n}}^{\mathrm{i}} \mathrm{Z}_{\mathrm{i}} \mathrm{N}_{\mathrm{i}}=\mathrm{F}\left(\mathrm{Z}_{1} \mathrm{~N}_{1}+\mathrm{Z}_{2} \mathrm{~N}_{2}\right)=\mathrm{i}_{\text {app }}
$$

To obtain an expression for the potential gradient in the electrolyte, Equation 13 can be used as:

$$
\frac{\mathrm{d} \phi}{\mathrm{dx}}=\frac{\mathrm{RT}\left[\left.\mathrm{F}\left(\mathrm{D}_{2}-\mathrm{D}_{1}\right) \frac{\partial \mathrm{c}}{\partial \mathrm{x}}\right|_{\mathrm{x}=0}-\mathrm{i}_{\text {app }}\right]}{\mathrm{F}^{2} \mathrm{c}\left(\mathrm{D}_{1}+\mathrm{D}_{2}\right)}
$$

The Equation 14, thus obtained, can be further substituted in Equation 10 as: 


$$
\frac{\partial c}{\partial t}=D_{\text {eff }} \frac{\partial^{2} c}{\partial x^{2}}
$$

The present model to simulate the electrodeposition of lithium on the metal electrode can be visualized as a moving boundary problem where the position of the interface of the electrode and electrolyte changes with time in the x-direction, mimicking the growth of the deposit on the surface.

For a constant value of current density, the velocity of the moving boundary is prescribed by Faraday's law ${ }^{17}$ as:

$$
\frac{\mathrm{ds}}{\mathrm{dt}}=\frac{\mathrm{i}_{\text {app }}}{\mathrm{F}} \frac{\mathrm{M}_{\mathrm{w}}}{\rho}
$$

where $s(m)$ is the length of the electrodeposit starting from the electrode/electrolyte interface at start of the charge cycle, $M_{w}$ is the molecular weight of lithium and $\rho$ is the density of the lithium deposit.

The boundary conditions for the ion concentration and potential across the interface are:

$$
\begin{array}{ll}
\text { At } x=0: & \left.\mathrm{N}_{1}\right|_{\mathrm{x}=0}=\frac{\mathrm{i}_{\text {app }}}{\mathrm{F}} \\
& \left.\mathrm{N}_{2}\right|_{\mathrm{x}=0}=0 \\
& \left.\mathrm{~N}_{1}\right|_{\mathrm{x}=\mathrm{L}}=\frac{\mathrm{i}_{\text {app }}}{\mathrm{F}} \\
& \left.\mathrm{N}_{2}\right|_{\mathrm{x}=\mathrm{L}}=0 \\
& \left.\phi\right|_{\mathrm{x}=\mathrm{L}}=0
\end{array}
$$


The anion is assumed not to react at either of the electrodes. In order to enhance the applicability of the present work and ease the computational effort, the length, time, and concentration are rendered dimensionless as follows:

$$
\mathrm{X}=\frac{\mathrm{x}}{\mathrm{L}}, \tau=\mathrm{t} \frac{\mathrm{D}_{\text {eff }}}{\mathrm{L}^{2}}, \mathrm{C}=\frac{\mathrm{c}}{\mathrm{c}_{0}}
$$

Thus, the velocity of the moving interface in the dimensionless form can now be expressed as:

$$
\frac{\mathrm{dS}}{\mathrm{d} \tau}=\frac{\mathrm{i}_{\mathrm{app}} \mathrm{L}}{\mathrm{FD}_{1} \mathrm{c}_{\mathrm{o}}} \frac{\mathrm{M}_{\mathrm{w}} \mathrm{c}_{0}}{\rho}=\delta \kappa
$$

Two additional dimensionless groups appear in Equation 17, namely, dimensionless current density, $\delta=\left(\frac{\mathrm{i}_{\text {app }} \mathrm{L}}{\mathrm{FD}_{1} \mathrm{c}_{0}}\right)$ and proportionality constant, $\kappa=\left(\frac{\mathrm{M}_{\mathrm{w}} \mathrm{c}_{0}}{\rho}\right)$.

This model assumes that the moving interface velocity is proportional to the applied current density. The ionic species concentration and the overpotential vary with position along the length of the cell. Since the interface moves, the position changes with time at a rate given by Equation 17. Equations 14 and 15 are thus transformed using Landau transform ${ }^{18}$ where the position along the length of the cell is redefined as:

$$
\mathrm{Z}=\frac{\mathrm{X}-\mathrm{S}(\tau)}{1-\mathrm{S}(\tau)}
$$

Using the transformed variable $Z$, the governing equation for the concentration, Equation 15, can be rewritten as:

$$
\frac{\partial \mathrm{C}}{\partial \tau}=\frac{1}{(1-\mathrm{S})^{2}} \frac{\partial^{2} \mathrm{C}}{\partial \mathrm{Z}^{2}}+\frac{\mathrm{dS}}{\mathrm{d} \tau} \frac{(1-\mathrm{Z})}{(1-\mathrm{S})} \frac{\partial \mathrm{C}}{\partial \mathrm{Z}}
$$


The transformed boundary conditions are:

At $Z=0:\left.\quad \frac{\partial C}{\partial Z}\right|_{Z=0}=\frac{\delta}{2}(1-S)$

At $Z=1:\left.\quad \frac{\partial C}{\partial Z}\right|_{Z=1}=\frac{\delta}{2}(1-S)$

In terms of $Z$, the equation for the potential (Equation 14) across the electrolyte can be rearranged

as:

$$
\frac{\frac{\mathrm{d} \phi}{\mathrm{dZ}}}{(1-\mathrm{S})}=\frac{\mathrm{RTL}}{\mathrm{CF}(1-\mathrm{S})}\left(\frac{\mathrm{D}_{2}-\mathrm{D}_{1}}{\mathrm{D}_{1}+\mathrm{D}_{2}}\right) \frac{\partial \mathrm{C}}{\partial \mathrm{Z}}-\frac{\mathrm{RT} \delta \mathrm{D}_{1}}{\mathrm{C}_{1} \mathrm{~F}\left(\mathrm{D}_{1}+\mathrm{D}_{2}\right)}
$$

The boundary condition for the potential $\phi$ is:

$$
\left.\phi\right|_{\mathrm{Z}=1}=0
$$

The initial conditions for the given system are considered as $C(Z, 0)=1$, and $\phi(Z, 0)=0$.

In this model, the deposition and dissolution reactions are defined by the Butler-Volmer kinetics:

$$
\mathrm{i}_{\text {app }}=\mathrm{i}_{0, \text { ref }}\left(\frac{\mathrm{c}_{1}(\mathrm{x}, \mathrm{t})}{\mathrm{c}_{\text {ref }}}\right)^{\alpha_{\mathrm{a}}}\left[\exp \left(\frac{\alpha_{\mathrm{a}} \mathrm{F} \eta(\mathrm{t})}{\mathrm{RT}}\right)-\exp \left(\frac{-\alpha_{\mathrm{c}} \mathrm{F} \eta(\mathrm{t})}{\mathrm{RT}}\right)\right]
$$

where the $i_{0, \text { ref }}$ is the effective exchange current density, $\alpha_{a}$ and $\alpha_{c}$ are the charge transfer coefficients of the anode and cathode, respectively. The kinetics used in the problem formulation (Equation 21) does not account for the changes in surface area associated with the morphology of the deposit.

Equation 21 can be rewritten in the dimensionless form as: 


$$
\delta=\delta_{\mathrm{o}}\left(\frac{\mathrm{C}(\mathrm{Z}, \tau) \mathrm{c}_{0}}{\mathrm{c}_{\mathrm{ref}}}\right)^{\alpha_{\mathrm{a}}}\left[\exp \left(\frac{\alpha_{\mathrm{a}} \mathrm{F} \eta(\tau)}{\mathrm{RT}}\right)-\exp \left(\frac{-\alpha_{\mathrm{c}} \mathrm{F} \eta(\tau)}{\mathrm{RT}}\right)\right]
$$

The partial differential equations (PDEs) for concentration and potential within the electrolyte obtained after Landau transformation, Equations 19 and 20, were discretized using finite difference method. Equations 23 and 24 show the discretized form for the transformed PDEs.

$$
\begin{gathered}
\frac{\partial \mathrm{C}_{\mathrm{i}}}{\partial \tau}=\frac{\mathrm{C}_{\mathrm{i}-1}-2 \mathrm{C}_{\mathrm{i}}+\mathrm{C}_{\mathrm{i}+1}}{\mathrm{~h}^{2}(1-\mathrm{S})^{2}}+\frac{1}{2} \frac{\mathrm{dS}}{\mathrm{d} \tau} \frac{(1-\mathrm{ih})}{1-\mathrm{S}} \frac{\mathrm{C}_{\mathrm{i}+1}-\mathrm{C}_{\mathrm{i}-1}}{\mathrm{~h}} \\
\frac{1}{2 \mathrm{~h}} \frac{\phi_{\mathrm{i}+1}-\phi_{\mathrm{i}-1}}{(1-\mathrm{S})}=\left(\frac{\mathrm{D}_{2}-\mathrm{D}_{1}}{\mathrm{D}_{1}+\mathrm{D}_{2}}\right) \frac{\left(\mathrm{C}_{\mathrm{i}+1}-\mathrm{C}_{\mathrm{i}-1}\right)}{2 \mathrm{~h}(1-\mathrm{S})} \frac{\mathrm{RTL}}{\mathrm{C}_{\mathrm{i}} \mathrm{F}}-\frac{\mathrm{RT} \delta \mathrm{D}_{1}}{\mathrm{C}_{\mathrm{i}} \mathrm{F}\left(\mathrm{D}_{1}+\mathrm{D}_{2}\right)}
\end{gathered}
$$

where i ranges from 1 to $\mathrm{N}$. The discretized boundary conditions for $\mathrm{C}$ and $\phi$ are also written below.

$$
\begin{gathered}
\frac{-\mathrm{C}_{2}-3 \mathrm{C}_{0}+4 \mathrm{C}_{1}}{2 \mathrm{~h}}=\frac{\delta}{2}(1-\mathrm{S}) \\
\frac{\mathrm{C}_{\mathrm{N}-1}+3 \mathrm{C}_{\mathrm{N}+1}-4 \mathrm{C}_{\mathrm{N}}}{2 \mathrm{~h}}=\frac{\delta}{2}(1-\mathrm{S}) \\
\phi_{\mathrm{N}+1}=0
\end{gathered}
$$

Equations 19-21 result in the set of differential algebraic equations (DAEs) of the model. The list of the pertinent parameters for this model is tabulated for reference (Table 1). The aforementioned discretized equations were solved using Maple's stiff solver aided by consistent initialization for algebraic variables approach as delineated by Lawder et al. ${ }^{19}$. The variation in the values for dimensionless concentration obtained at dimensionless time, $\tau=3$ is shown in Table 2 along with absolute error between the solutions obtained with the new and old $\mathrm{h}$ values, $\mathrm{e}(\mathrm{h})$, and the order of convergence for decreasing values of the grid spacing, $\mathrm{h}$. For $\mathrm{h}=1 / 32$, the CPU time taken to solve 
the discretized set of equations is 188 milliseconds while the same takes 32 milliseconds with IDA solver in C platform. An error analysis was performed by doubling the number of nodes. It is important to observe that second order method provides a second order of convergence as obtained in Table 2.

\section{Results and Discussion}

This section examines the one-dimensional transport model formulated to resolve the effects of diffusion and lithium ion transport on the deposition of lithium leading to the formation of dendrites on the electrode surface. The deposition of lithium is inscribed as inverse signatures onto the voltage response of a cell designed with a lithium metal anode. This characteristic behavior, the so-called inverse signature, is highlighted in the red circle in Figure 2 for a $\mathrm{Li}-\mathrm{Cu}$ electrochemical cell ${ }^{16}$ as an illustration. Such inverse signatures are observed due to the balance between the motion of the interface and the diffusion of lithium. The moving boundary model can capture this balance and predict inverse signatures due to the transport mechanisms that lead to deposition. The kinetics used to describe the deposition is not modified to account for the inverse signature. The ability of the current model to produce inverse signatures is demonstrated below for the aforementioned ranges of the values of current density, diffusivities of the ionic species and $\kappa$.

\section{Effect of Current Density}

Chazaviel ${ }^{7}$ postulated that the lithium protrusions appear due to the formation of a space charge region at the electrode/electrolyte interface where dendrite growth takes place, which occurs when the anions in this region are depleted. This particularly occurs when the values of current density exceed the mass transfer limiting current $\left(i_{m}\right)$, i.e., when the salt concentration at the 
electrode/electrolyte interface reaches zero. ${ }^{20}$ At these higher current densities $\left(>\mathrm{i}_{\mathrm{m}}\right)$, the growth is diffusion-limited..$^{5,11,10}$

The model proposed by Chazaviel ${ }^{7}$ to calculate the growth velocity does not apply at lower current densities $\left(<\mathrm{i}_{\mathrm{m}}\right)$. To explain the formation of dendrites at these current densities, Orsini et al. ${ }^{21}$ observed the nature of the deposit using Scanning Electron Microscopy (SEM) images. It was observed that the deposits are mossy at lower charge current densities unlike the dendrites observed at higher current densities associated with diffusion-limited growth. This implies the formation of lithium deposits to be surface controlled at lower current densities, with short range diffusion of $\mathrm{Li}^{+}$ions occurring within the SEI layer on the electrode surface. ${ }^{22}$

The current model equations have been evaluated for concentration and potential profiles in the electrolyte domain for different values of current densities, expressed as fractions of the mass transfer limiting current density, $\mathrm{i}_{\mathrm{m}}$. Figure 3(a) shows the plots for the variation of concentration with time at the electrode/electrolyte interface of the symmetric cell for different values of current densities. For smaller values of current density, the diffusion of lithium is expected to be faster which is manifested in terms of smaller gradients and relatively flatter concentration profiles. Under these circumstances, the diffusion of $\mathrm{Li}^{+}$ions reaches steady state and the rate of lithium deposition or the moving boundary velocity is slow. As the fraction of $i_{m}$ increases in current density, the concentration profile dips and rises as the interface shifts towards higher electrolyte concentrations. The dip in concentration occurs as the salt at the interface is depleted, and the rise occurs as deposition takes place, increasing the $\mathrm{Li}^{+}$concentration at the interface as it moves.

The overpotential profiles mimic the concentration profile as seen in Figure 3(b). A higher magnitude of overpotential is required to drive the deposition reaction at higher values of current density. As the current density increases, a sharp dip in overpotential is realized, in-line with the 
trend observed with the lithium ion concentration at the interface. This results in the unique inverse signatures as the overpotential reverses its slope with the increase in the rate of deposition on the surface of the electrode. This signature is observed as the deposit encounters higher localized concentrations of salt in the electrolyte, with the velocity of the moving boundary increasing and the deposit moving towards the bulk electrolyte.

These signatures are produced due to the influence of the applied current density on the concentration gradient that drives transport of lithium ions, and the motion of the interface. Inverse signatures can also be kinetically explained with the competing mechanisms of lithium deposition and dissolution. . These mechanisms are described in the effective exchange current density in the Butler-Volmer kinetics. The effective exchange current density is a function of the exchange current densities of the 'fast' and 'slow' reactions that correspond to dendrite growth and lithium dissolution from the bulk. ${ }^{9,16}$ The effect of the reference exchange current density in dimensionless form used in the Butler-Volmer kinetic expression is shown in Figure 4. The overpotential response for different values of $\delta_{0}$ is shown in Figure 4. The concentration profiles Figure 4(a) overlap each other, suggesting that the balance between the $\mathrm{Li}^{+}$ion diffusion and the motion of the interface is independent of the reference exchange current density. The dip and rise in the overpotential profiles remains the same for different $\delta_{0}$ values are parallel to each other This can infer that the model predicts the inverse signatures due to the transport mechanisms of the lithium ions which inherently leads to electrodeposition and dendrite formation and without the actual modification of the reference exchange current density to reflect the deposition and dissolution mechanisms. 


\section{Effect of Ion Diffusivities on Lithium Deposition}

For a given current density, the diffusivity of lithium ions in the electrolyte can influence the motion of the interface that impacts the formation of voltage signatures. In order to delineate the contribution of each of the ionic species on deposition, a constant $\beta$ has been defined as the ratio of the anionic diffusivity to the cationic diffusivity.

$$
\beta=\frac{D_{2}}{D_{1}}
$$

The influence of the transport properties of one ion over the other can be examined for their contribution on the formation of inverse voltage signatures and to access the effect of the transport of the ions on the overall deposition reaction seen on the electrode surface. The effective diffusivity $D_{\text {eff }}$ is a measure of this influence.

Figure 5 shows the concentration and overpotential profiles at a current density of $0.25 \mathrm{i}_{\mathrm{m}}$, which corresponds to the mass transfer limiting current density at $\beta=4$. At higher values of $\beta$, the diffusion is faster, the profiles stay flat and the concentration reaches steady state without the movement of the boundary to higher concentration regions. With more resistance to diffusion as $\mathrm{D}_{1}$ decreases, the presence of inverse signature intensifies as seen in Figure 5(b). The slower diffusion at low values of $\beta$ decreases the concentration and increases the overpotential to allow for the boundary to encounter higher concentration as it moves. Higher effective diffusivity results in faster lithium diffusion, and stronger inverse signatures are observed at lower values of values of $\mathrm{D}_{\mathrm{eff}}$. 


\section{Effect of Deposit Density}

The present model can also qualitatively predict unique inverse signatures which are observed due to the differences in the morphology of the deposit. The concentration profiles obtained on increasing the proportionality constant $\kappa$, related to the properties of lithium is shown in Figure 6(a). For a given value of current density, $\kappa$ contributes to the increase in moving boundary velocity. For a fixed initial salt concentration, when $\kappa$ is increased the density changes to a fraction $\varepsilon$ of the density of pure lithium, providing a rough indication that the deposit is more porous in nature. This effect can be seen in the concentration profiles and overpotential signatures as a more porous deposit shifts the boundary further into the electrolyte, as the volume occupied by a fixed mass of the deposit is higher. For higher values of $\kappa$, the value of $\varepsilon$ decreases, indicating that the nature of the deposit is less dense. This less dense deposit can comprise of dendritic structures, that protrude and grow from the tip, whereas a denser deposit can indicate that its morphology is mossy in nature.

From Figure 6(a), the dip and rise in concentration is steep and increases significantly for lower values of $\varepsilon$. The magnitude of the overpotential inverse signature decreases with decrease in $\varepsilon$ as seen in Figure 6(b), suggesting that for a given applied current density, dense deposits have a signature with a lower activation barrier. The strength of the inverse signature remains the same for each of the cases. These details regarding the morphology of the deposit can be further explored and substantiated by extending the current model to two dimensions which forms the course of the future work in this field.

Finally, the effect of the significant dimensionless groups spanned in this study on the inverse signatures is summarized in Table 3 . The influence of cell design parameters on the strength of the inverse signature is shown in Figure 7. Inverse signatures are observed for lower values of the 
initial salt concentration as seen in Figure 7(a) and 7(b). Figure 7(a) shows the normalized concentration profiles (the concentration at the moving interface divided by the initial concentration) for different values of the initial salt concentration. The dip and rise in concentration is steep for lower initial salt concentrations. This trend indicates the motion of the interface is faster for lower concentrations, and the diffusion of lithium ions reaches steady state for high salt concentrations. Figure 7(b) shows that the inverse signature is strong for lower concentrations. Several studies have been conducted to examine if highly concentrated electrolytes have an effect on dendrite formation ${ }^{3,15,23}$. Increasing the salt concentration in the electrolyte increases the density of lithium, resulting in mossy-like or suppressed dendritic deposition on the electrode surface. ${ }^{15}$ This can be observed in Figure 6, where higher values of $\varepsilon$ show a weaker concentration signature and a higher activation barrier for the motion of the interface. The lack of inverse signatures indicates that the motion of the interface is suppressed for higher salt concentrations.

It can be added here that cells exhibit local variations in current density and concentration at the metal electrode surface. These variations result in the inverse signatures seen in the voltage response of lithium-metal batteries. For example, inverse signatures can sometimes be observed due to non-uniform current distribution. The present study is conducted using a one-dimensional model which depicts that the inverse signatures due to transport are prominent at high current densities close to the mass transfer limiting current density, $\mathrm{i}_{\mathrm{m}}$. These currents are seen in real cells that show local inhomogeneities in current density. A two-dimensional model can further capture the effect of these variations on inverse signatures.

The signatures produced are captured due to the transport of ions during the electrodeposition of lithium. The model used in this work is a symmetric cell with a large separation between the electrodes. The diffusion time scale for this cell is large, which leads to the motion of the interface 
to be dominant. This causes the inverse signature to be pronounced. In a real cell, the thickness of the separator is much smaller, and the formation of transport induced signatures is masked as the $\mathrm{Li}^{+}$ion concentration reaches steady state. Figures $7(\mathrm{c})$ and $7(\mathrm{~d})$ show the effect of the cell dimensions observed in real lithium-metal cells vs. symmetric cells. The inverse signature observed from the symmetric cell in this work is more notable compared to typical lithium metal batteries. The strength of inverse signatures from the 'transport' only model is more prevalent with larger symmetric cells. While these cells are useful to understand the mechanisms of deposition and dendrite formation, inverse signatures formed due to lithium transport are more pronounced in symmetric cells. A combination of the kinetics of the lithium electrodeposition process and accounting for the local inhomogeneities may predict these signatures for real lithium-metal cells.

\section{Conclusions}

In this paper, a simple one-dimensional moving boundary model is presented which predicts the characteristic voltage signatures that arise due to the deposition of lithium on the electrode surface without the need for a modified form of kinetics to accommodate for the different reaction pathways which occur during the electrodeposition of lithium. These voltage signatures are predominant with the increase in applied current density as the deposition becomes diffusion limiting and the motion of the interface increases. The diffusivity of the individual ionic species plays a role in observing unique inverse signatures, where smaller cation diffusivities showed prominent signatures. The study of the individual ionic species provides an understanding to the nature of the deposit, compared to observing the result from adjusting the effective diffusivity.

The key feature of the current model can be attributed to the fact that it only includes the transport of lithium ions and the interface of the deposited lithium is modeled as a moving boundary. The model was tested across different design parameters that are known to influence the nucleation 
and growth of the deposits. This simple one-dimensional transport model could successfully capture the inverse signatures. These inverse signatures are predicted with equations that correspond to the transport of lithium and are more prominent in thick symmetric cells compared to thin lithium metal batteries.

Extending this model into a two-dimensional space to capture the effects of localized current densities, shape of the deposit and the effect of pressure on the dendrite growth is necessary to accurately model lithium deposition.

The model is available as executable code, soon to be hosted at the corresponding author's website (http:/sites.utexas.edu/maple).

\section{Acknowledgements}

The authors would like to express gratitude to Assistant Secretary for Energy Efficiency and Renewable Energy, Office of Vehicle Technologies of the DOE through the Advanced Battery Material Research (BMR) Program (Battery500 consortium). Part of the moving boundary work

for this model at the University of Texas at Austin was supported by U.S. DOE Office of Electricity award DEAC05-76RL01830 through PNNL subcontract 475525. 


\section{References}

1. W. Xu, J. Wang, F. Ding, X. Chen, E. Nasybulin, Y. Zhang, and J. G. Zhang, Energy Environ. Sci., 7, 513 (2014).

2. D. Lin, Y. Liu, and Y. Cui, Nat. Nanotechnol., 12, 194 (2017).

3. J. Qian, W. A. Henderson, W. Xu, P. Bhattacharya, M. Engelhard, O. Borodin, and J. G. Zhang, Nat. Commun., 6, 6362 (2015).

4. J. I. Yamaki, S. I. Tobishima, K. Hayashi, K. Saito, Y. Nemoto, and M. Arakawa, J. Power Sources, 74, 219 (1998).

5. T. A. Witten and L. M. Sander, Phys. Rev. B, 27, 240 (1983).

6. J. L. Barton and J. O. Bockris, Proc. R. Soc. A, 268, 485 (1961).

7. J. N. Chazalviel, Phys. Rev. A, 42, 7355 (1990).

8. C. Monroe and J. Newman, J. Electrochem. Soc., 150, A1377 (2003).

9. K. N. Wood, E. Kazyak, A. F. Chadwick, K. H. Chen, J. G. Zhang, K. Thornton, and N. P. Dasgupta, ACS Cent. Sci., 2, 790 (2016).

10. K. N. Wood, M. Noked, and N. P. Dasgupta, ACS Energy Lett., 2, 664 (2017).

11. P. Bai, J. Li, F. R. Brushett, and M. Z. Bazant, Energy Environ. Sci., 9, 3221 (2016).

12. M. Arakawa, S. Tobishima, Y. Nemoto, M. Ichimura, and J. Yamaki, J. Power Sources, 43, 27 (1993).

13. C. Brissot, M. Rosso, J. N. Chazalviel, and S. Lascaud, J. Power Sources, 81-82, 925 (1999). 
14. L. Frenck, G. K. Sethi, J. A. Maslyn, and N. P. Balsara, Front. Energy Res., 7, 115 (2019).

15. S. Lv, T. Verhallen, A. Vasileiadis, F. Ooms, Y. Xu, Z. Li, Z. Li, and M. Wagemaker, Nat. Commun., 9, 2152 (2018).

16. A. Subramaniam, J. Chen, T. Jang, R. M. Kasse, M. F. Toney, and N. R. Geise, J. Electrochem. Soc., 166, A3806 (2019).

17. R. Alkire, T. Bergh, and R. L. Sani, J. Electrochem. Soc., 125, 1981 (1978).

18. H. G. Landau, Q. Appl. Math., 8, 81 (1950).

19. M. T. Lawder, V. Ramadesigan, B. Suthar, and V. R. Subramanian, Comput. Chem. Eng., 82, $283(2015)$

20. H. J. S. Sand, London, Edinburgh, Dublin Philos. Mag. J. Sci., 1, 45 (1901).

21. F. Orsini, A. Du Pasquier, B. Beaudouin, J. M. Tarascon, M. Trentin, N. Langenhuizen, E. De Beer, and P. Notten, J. Power Sources, 81-82, 918-921 (1999).

22. X. Cheng, R. Zhang, C. Zhao, and Q. Zhang, Chem. Rev., 117, 10403 (2017).

23. S.-K. Jeong, H.-Y. Seo, D.-H. Kim, H.-K. Han, J.-G. Kim, Y. B. Lee, Y. Iriyama, T. Abe, and Z. Ogumi, Electrochem. commun., 10, 635 (2008). 


\section{List of Figures}

Figure 1. Schematic of the lithium symmetric cell showing the moving boundary $s(t)$ in the $x-$ direction.

Figure 2. The voltage profile obtained from a $\mathrm{Li}-\mathrm{Cu}$ electrochemical cell with the inverse signature circled in red $^{16}$.

Figure 3. Variation of (a) concentration and (b) interfacial overpotential with time at the electrode/electrolyte moving interface for different values of applied current densities with the corresponding values of the dimensionless group $\delta$.

Figure 4. Variation of (a) concentration and (b) interfacial overpotential with time at the electrode/electrolyte moving interface for different values of dimensionless exchange current densities.

Figure 5. Variation of (a) concentration and (b) interfacial overpotential with time at the electrode/electrolyte moving interface for different values of $\mathrm{D}_{\text {eff }}$ at $0.25 \mathrm{i}_{\mathrm{m}}$ with the corresponding values of the dimensionless group $\beta$.

Figure 6. Variation of (a) concentration and (b) interfacial overpotential with time at the electrode/electrolyte moving interface for different values of $\varepsilon$ at $i_{m}$ at a constant initial concentration with the corresponding values of the dimensionless group $\kappa$.

Figure 7. Variation of (a) concentration and (b) with time for different electrolyte concentrations at $\mathrm{i}_{\mathrm{m}} / 2$; Variation of (c) concentration and (d) interfacial overpotential with time for different cell dimensions at $\mathrm{i}_{\mathrm{m}}$. 


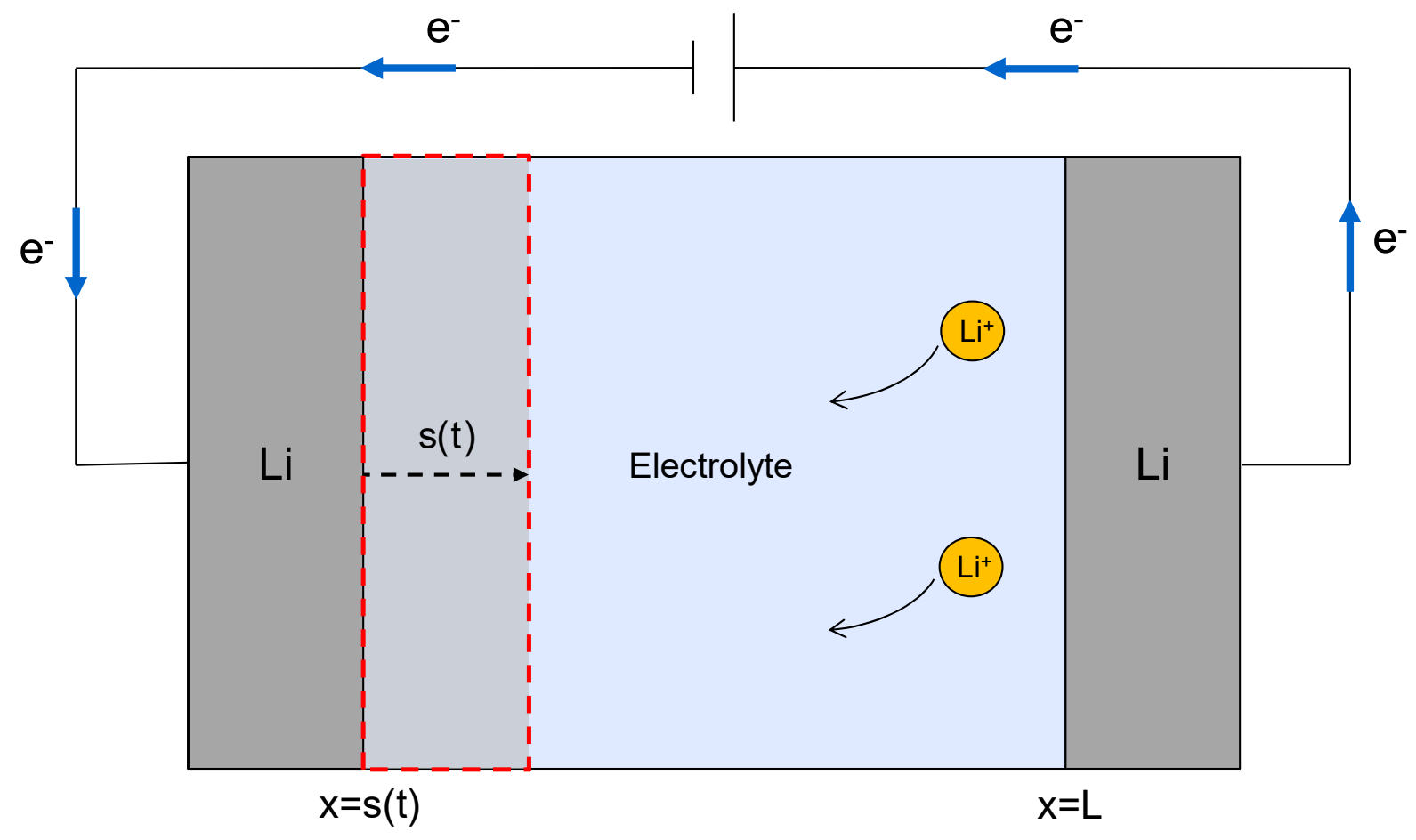

Figure 1. Schematic of the lithium symmetric cell showing the moving boundary $s(t)$ in the $\mathrm{x}$-direction. 


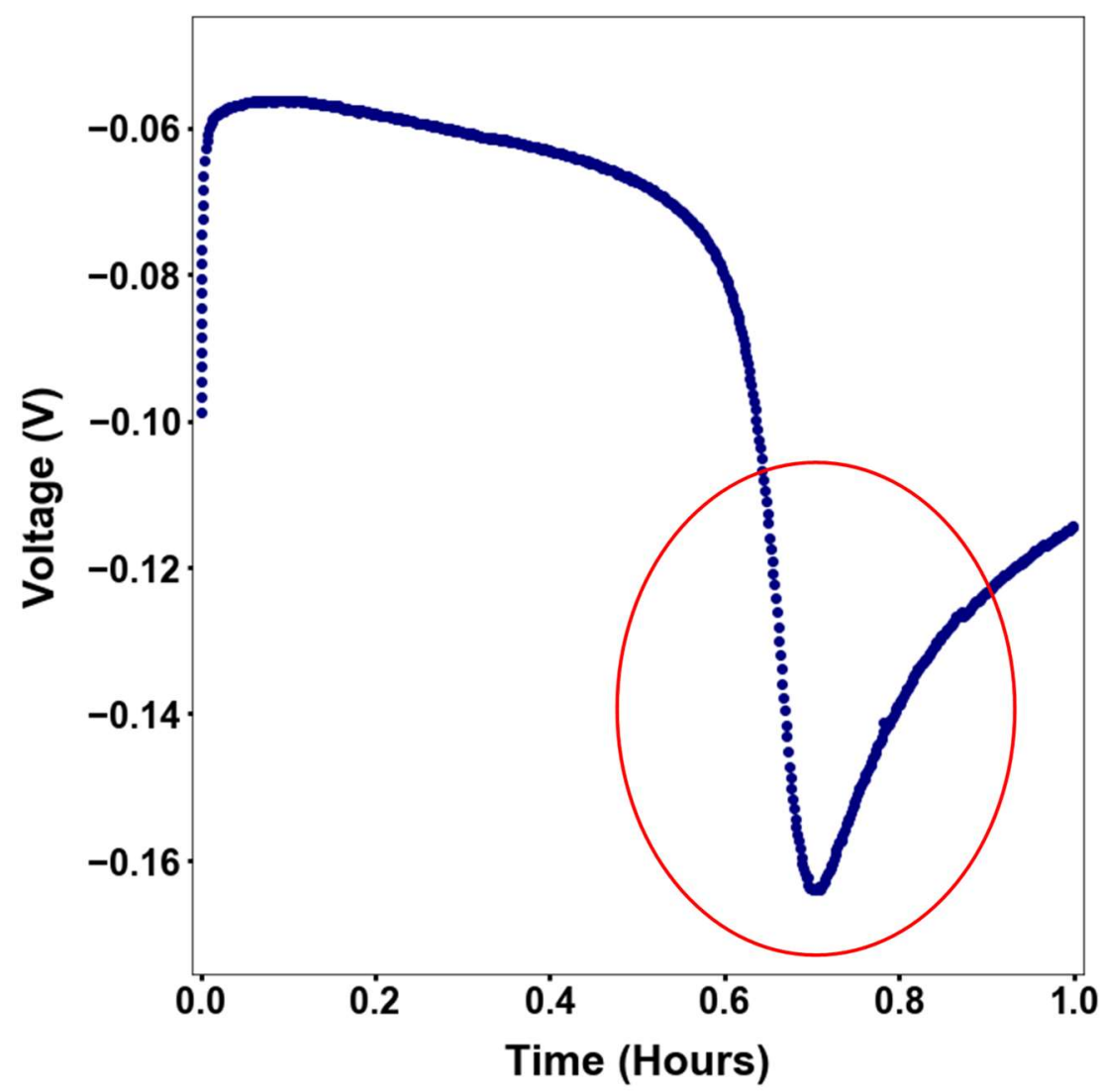

Figure 2. The voltage profile obtained from a $\mathrm{Li}-\mathrm{Cu}$ electrochemical cell with the inverse signature circled in $\operatorname{red}^{16}$. 

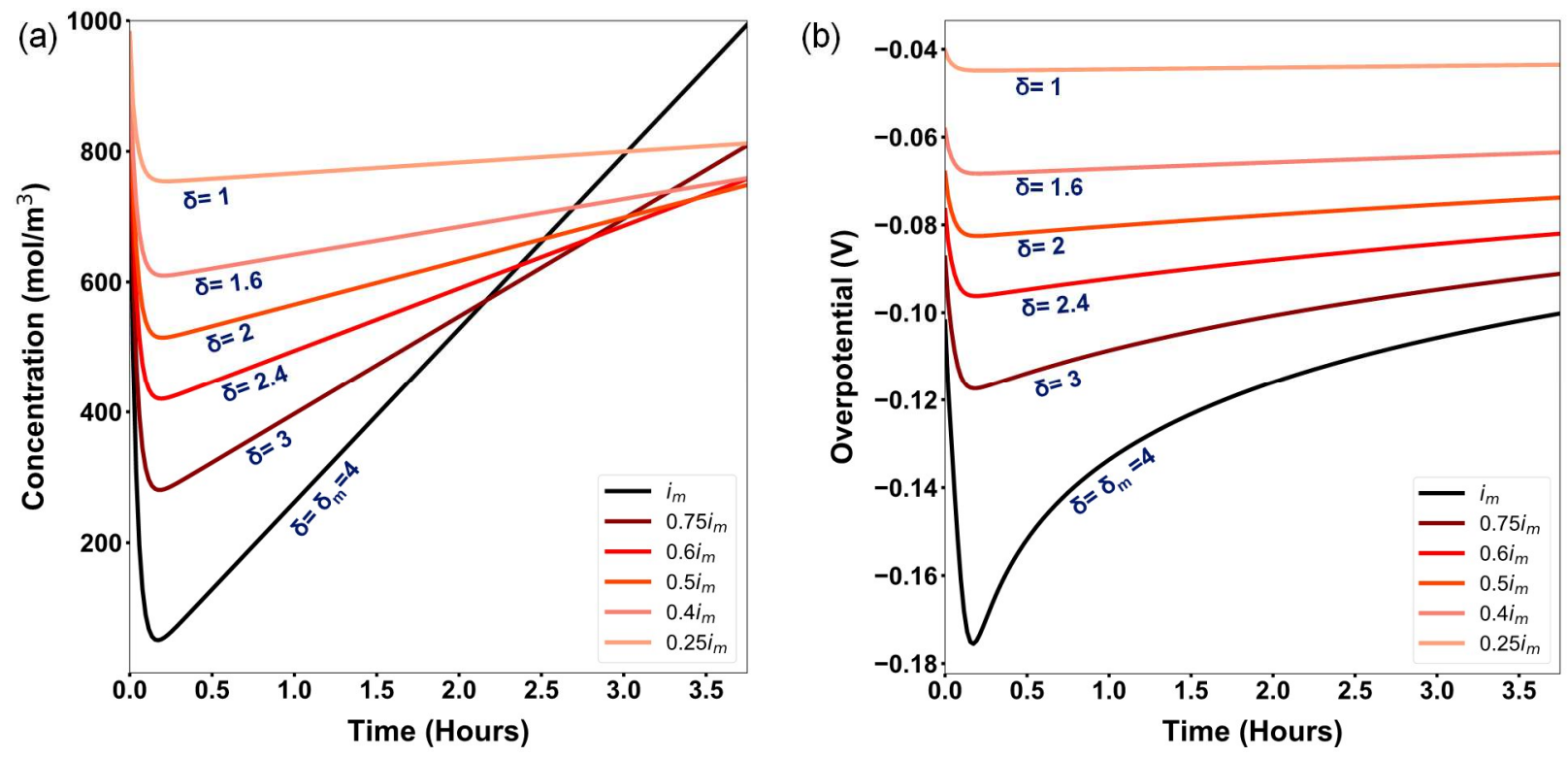

Figure 3. Variation of (a) concentration and (b) interfacial overpotential with time at the electrode/electrolyte moving interface for different values of applied current densities with the corresponding values of the dimensionless group $\delta$. 

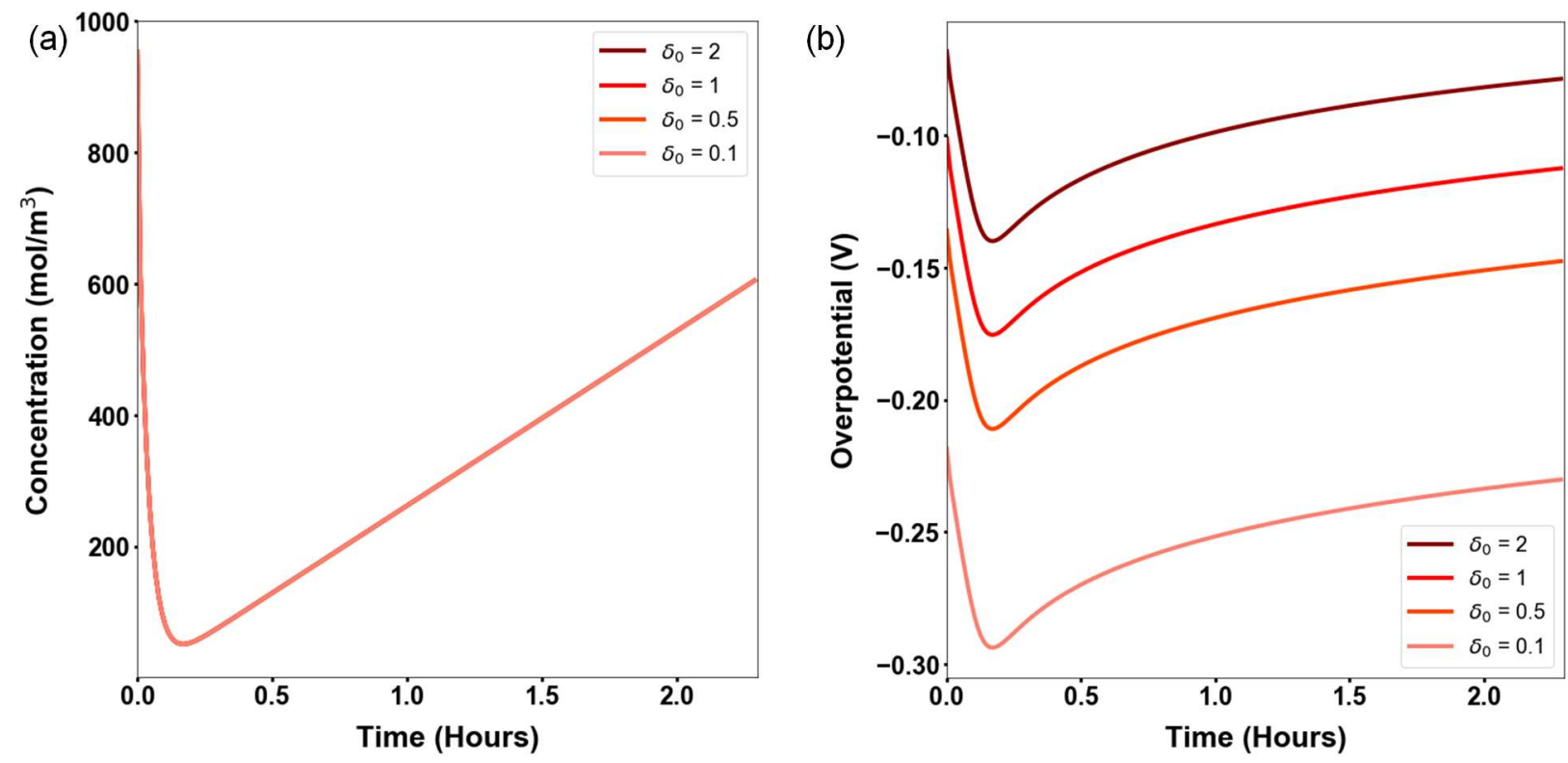

Figure 4. Variation of (a) concentration and (b) interfacial overpotential with time at the electrode/electrolyte moving interface for different values of dimensionless exchange current densities $\delta_{0}$. 

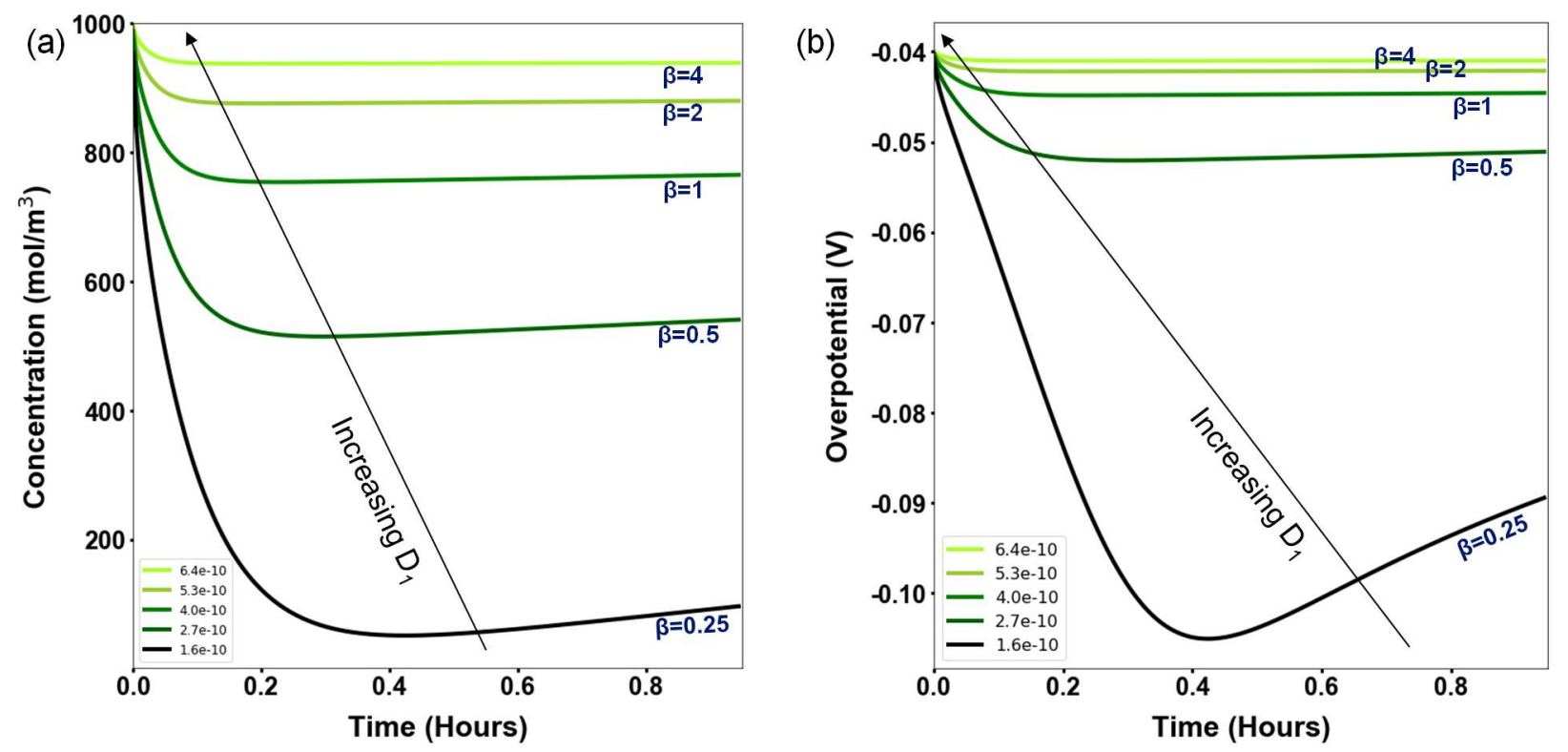

Figure 5. Variation of (a) concentration and (b) interfacial overpotential with time at the electrode/electrolyte moving interface for different values of $D_{\text {eff }}$ at $0.25 \mathrm{i}_{\mathrm{m}}$ with the corresponding values of the dimensionless group $\beta$. 

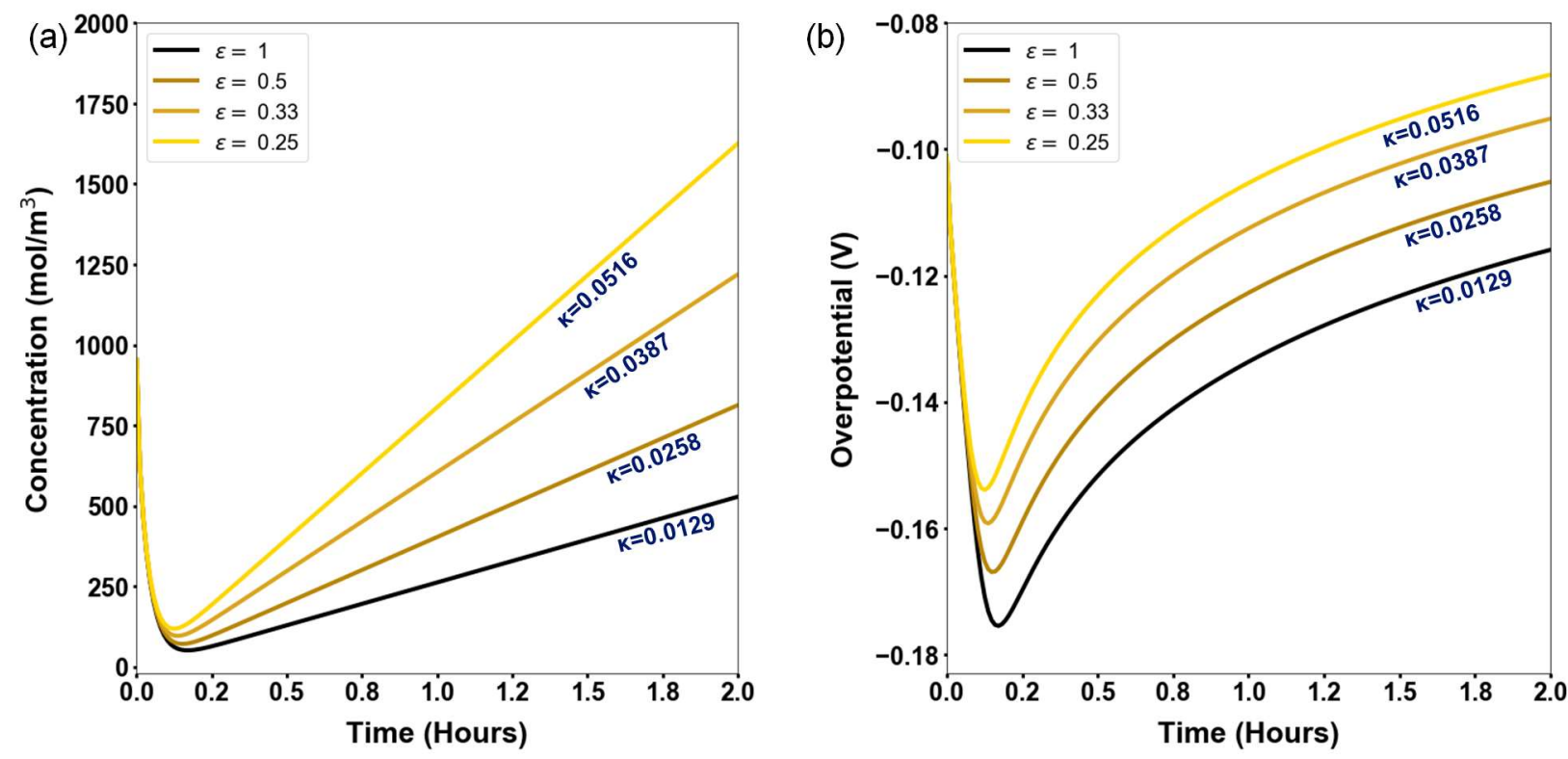

Figure 6. Variation of (a) concentration and (b) interfacial overpotential with time at the electrode/electrolyte moving interface for different values of $\varepsilon$ at im at a constant initial concentration with the corresponding values of the dimensionless group $\kappa$. 

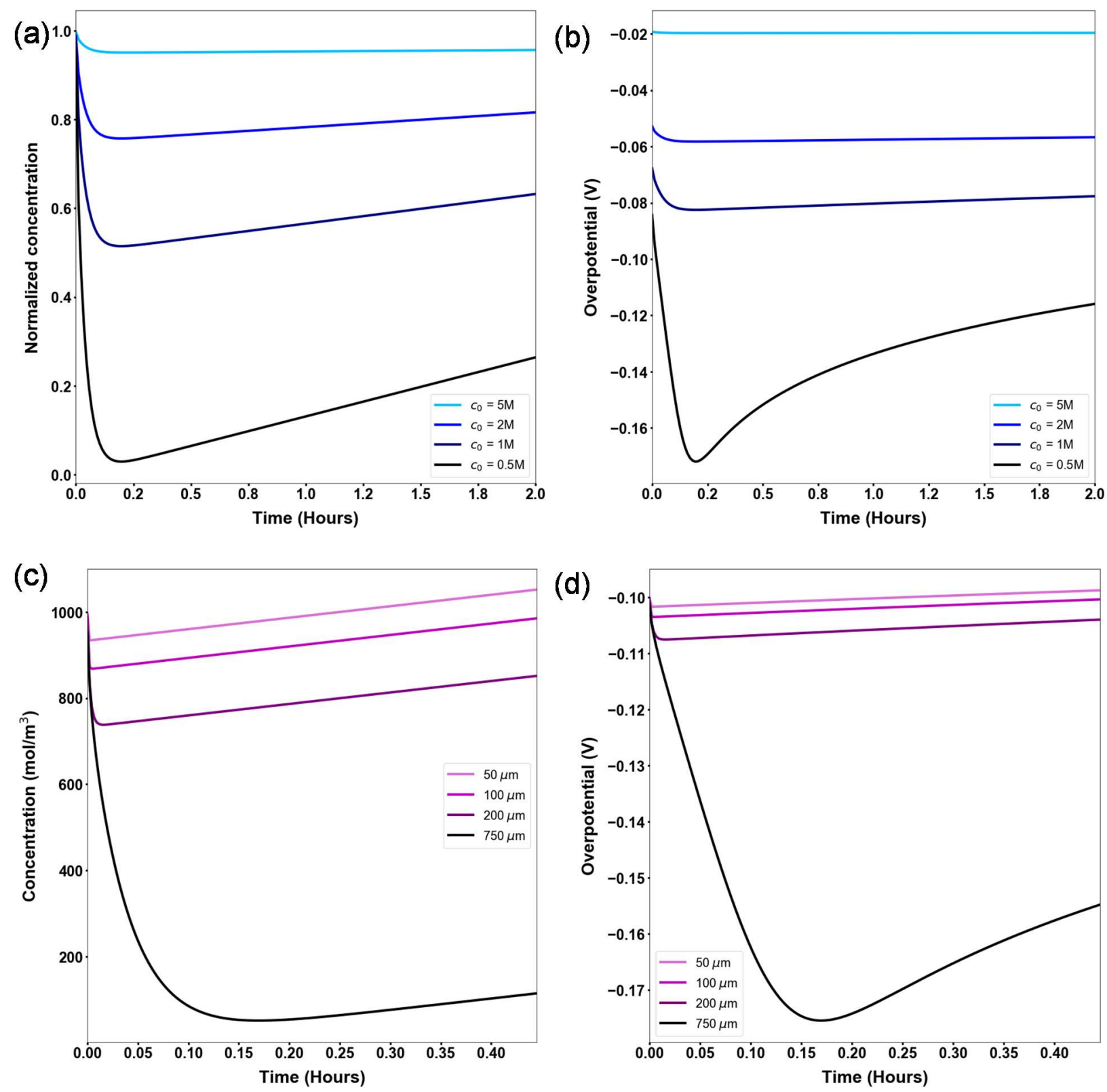

Figure 7. Variation of (a) concentration and (b) with time for different electrolyte concentrations at $\mathrm{i}_{\mathrm{m}} / 2$; Variation of (c) concentration and (d) interfacial overpotential with time for different cell dimensions at $i_{m}$. 


\section{List of Tables}

Table 1. List of base case parameters used in the model.

Table 2. Error and convergence analysis for the model using dimensionless concentration at the electrode surface $(\mathrm{X}=0)$ at $\tau=3$.

Table 3. Summary of the inverse signature trends observed from the present model for different dimensionless groups. 


\begin{tabular}{lccc}
\hline \multicolumn{1}{c}{ Table 1. List of base case parameters used in the model. } & \\
\hline Parameter & Symbol & Value & Units \\
\hline Initial concentration & $\mathrm{c}_{0}$ & 1000 & $\mathrm{~mol} / \mathrm{m}^{3}$ \\
Cell dimension & $\mathrm{L}$ & $7.5 \times 10^{-4}$ & $\mathrm{~m}$ \\
Temperature & $\mathrm{T}$ & 298 & $\mathrm{~K}$ \\
Universal gas constant & $\mathrm{R}$ & 8.314 & $\mathrm{~J} / \mathrm{mol} \mathrm{K}$ \\
Faraday's constant & $\mathrm{F}$ & 96487 & $\mathrm{C} / \mathrm{mol}$ \\
Li ${ }^{+}$diffusivity & $\mathrm{D}_{1}$ & $4 \times 10^{-10}$ & $\mathrm{~m}{ }^{2} / \mathrm{sec}$ \\
Anion diffusivity & $\mathrm{D}_{2}$ & $4 \times 10^{-10}$ & $\mathrm{~m}{ }^{2} / \mathrm{sec}$ \\
Reference concentration & $\mathrm{c}_{\text {ref }}$ & 1000 & $\mathrm{~mol} / \mathrm{m}^{3}$ \\
Exchange current density & $\mathrm{i}_{0, \text { ref }}$ & 30 & $\mathrm{~A} / \mathrm{m}^{2}$ \\
Molecular weight of lithium & $\mathrm{M}_{\mathrm{W}}$ & 0.006941 & $\mathrm{~kg} / \mathrm{mol}$ \\
Density of lithium metal & $\rho$ & 534 & $\mathrm{~kg} / \mathrm{m}^{3}$ \\
\hline
\end{tabular}


Table 2. Error and convergence analysis for the model using dimensionless concentration at the electrode surface $(X=0)$ at $\tau=3$.

\begin{tabular}{cccc}
$\begin{array}{c}\text { Grid } \\
\text { spacing } \\
\mathbf{h}\end{array}$ & $\begin{array}{c}\text { Dimensionless } \\
\mathbf{c o n c e n t r a t i o n} \\
\mathbf{C}(\mathbf{X}=\mathbf{0}, \boldsymbol{\tau}=\mathbf{3})\end{array}$ & $\begin{array}{c}\text { Absolute error } \\
\mathbf{e}(\mathbf{h}) \mid\end{array}$ & $\begin{array}{c}\text { Order of convergence } \\
\log _{2}\left(\frac{\mathbf{e}(\mathbf{h})}{\mathbf{e}(\mathbf{h} / \mathbf{2})}\right)\end{array}$ \\
\hline $1 / 2$ & 0.768571279086743 & $2.26812345 \times 10^{-4}$ & 2.70986481416186 \\
$1 / 4$ & 0.768344466741563 & $3.46669869 \times 10^{-5}$ & 2.56143850913872 \\
$1 / 8$ & 0.768309799754704 & $5.87281435 \times 10^{-6}$ & 2.40292748076210 \\
$1 / 16$ & 0.768303926940350 & $1.11043470 \times 10^{-6}$ & 2.25801207128913 \\
$1 / 32$ & 0.768302816505653 & $0.23214731 \times 10^{-7}$ & 2.14967537139309 \\
$1 / 64$ & 0.768302584358341 & $0.52317489 \times 10^{-8}$ & - \\
$1 / 128$ & 0.768302532040852 & - & - \\
\hline
\end{tabular}




\begin{tabular}{ccc}
\hline $\begin{array}{c}\text { Table 3. Summary of the inverse signature trends observed from the present model for } \\
\text { different dimensionless groups. }\end{array}$ \\
$\begin{array}{c}\text { Dimensionless } \\
\text { Group }\end{array}$ & Range of values & Nature of inverse signature \\
\hline & $\delta<2$ & Not observable \\
$\delta$ & $2<\delta<2.4$ & Weak \\
& $\delta>2.4$ & Strong \\
$\beta$ & $\beta<4$ & Not observable \\
$\kappa$ & $\beta>4$ & Strong \\
\end{tabular}

